\title{
Análise da qualidade de vida após a alta hospitalar em sobreviventes de sepse grave e choque séptico
}

\author{
Glauco Adrieno Westphal, ${ }^{1}$ Kalinca Daberkow Vieira, ${ }^{1}$ \\ Roman Orzechowski, Keitiane Michele Kaefer, ${ }^{1}$ \\ Viviane Renata Zaclikevis ${ }^{1}$ e Marco Fabio Mastroeni ${ }^{2}$
}

Como citar Westphal GA, Vieira KD, Orzechowski R, Kaefer KM, Zaclikevis VR, Mastroeni MF. Análise da qualidade de vida após a alta hospitalar em sobreviventes de sepse grave e choque séptico. Rev Panam Salud Publica. 2012;31(6):499-505.

RESUMO Objetivo. Descrever a repercussão da sepse grave e do choque séptico sobre a qualidade de vida após a alta hospitalar.

Métodos. Estudo controlado realizado em dois hospitais gerais de Joinville, Santa Catarina, Brasil, envolvendo pacientes internados com sepse grave ou choque séptico no período de agosto de 2005 a novembro de 2007. Os pacientes foram contatados por telefone entre junho e novembro de 2009. Os sobreviventes responderam ao Short Form-36, um questionário de qualidade de vida, dois anos após a alta. O questionário também foi respondido por um grupo controle composto de pessoas que habitavam o mesmo domicilio dos sobreviventes, sem internação recente e com idade mais próxima possível à do paciente.

Resultados. De 217 pacientes com sepse grave ou choque séptico, 112 (51,6\%) sobreviveram à internação. A sobrevida pós-alta hospitalar foi de 41,02\% em 180 dias, 37,4\% após um ano, 34,3\% em 18 meses e 32,3\% em dois anos. Trinta e seis sobreviventes responderam ao Short Form-36. Houve comprometimento da qualidade de vida dos sobreviventes (No. = 36) em relação ao grupo controle (No. $=36)$ nos domínios: capacidade funcional (59 \pm 32 versus $91 \pm 18 ; \mathrm{P}<0,001)$, vitalidade $(48 \pm 13$ versus $59 \pm 14 ; \mathrm{P}<0,008)$, saúde mental $(48 \pm 13$ versus $59 \pm 14 ; \mathrm{P}<0,03)$, dor $(50 \pm 26$ versus $76 \pm 16 ; \mathrm{P}<0,001)$, estado geral de saúde $(53 \pm 18$ versus $67 \pm 13 ; \mathrm{P}<0,004)$, aspectos físicos $(67 \pm 45$ versus $85 \pm 34 ; \mathrm{P}<0,05) e$ aspectos sociais $(70 \pm 28$ versus $90 \pm 16 ; \mathrm{P}<0,05)$.

Conclusões. Sepse grave ou choque séptico podem resultar em comprometimento significativo da qualidade de vida, assim como limitar a probabilidade de sobrevida a longo prazo.

Palavras-chave Sepse; choque séptico qualidade de vida; mortalidade; Brasil.

Sepse grave e choque séptico são as principais causas de morte nos pacientes internados em Unidades de Terapia Intensiva (UTIs) (1-3). Nos Estados Uni-

\footnotetext{
1 Hospital Municipal São José, Joinville, Santa Catarina, Brasil. Enviar correspondência para Kalinca Daberkow Vieira, kalincavieira@yahoo.com.br

2 Universidade de Joinville, Joinville, Santa Catarina, Brasil.
}

dos, a mortalidade hospitalar associada à sepse grave é de aproximadamente $25 \%$ dos pacientes internados (4), configurando a $13^{\mathrm{a}}$ causa de morte naquele país (5). Estudos epidemiológicos brasileiros demonstraram que esse número é ainda maior no Brasil, observando-se taxas de mortalidade ao $28^{\circ}$ dia de internação de até $57,1 \%$ dos pacientes $(6,7)$. A avaliação de dois anos após a Campanha de Sobrevivência à Sepse (CSS) evidencia clara redução da média mundial da mortalidade hospitalar associada à sepse ( $37 \%$ versus $30,8 \%$; $P<0,001$ ) (8). No Brasil, no entanto, essa taxa ainda é de 55,6\% entre os hospitais participantes da CSS (9). Além disso, é sabido que a alta letalidade da sepse não se restringe à fase 
aguda da doença, mas também aumenta o risco de morte ao longo dos anos subsequentes à alta hospitalar $(2,10)$.

E possível considerar que as falhas estruturais e organizacionais que resultam em taxas inaceitáveis de mortalidade associada à sepse nos países em desenvolvimento (11) também tenham implicações na qualidade de vida dos sobreviventes. Como a letalidade a curto e a longo prazo relacionada à sepse parece estar diretamente associada às disfunções orgânicas desenvolvidas na fase aguda, infere-se que tais disfunções podem implicar também no comprometimento crônico de capacidades físicas e mentais, que deterioram a qualidade de vida e a aptidão para o trabalho dos sobreviventes $(2,12)$.

$\mathrm{O}$ impacto das doenças sobre a qualidade de vida é avaliado com a aferição de variáveis que compõem o quotidiano humano, por meio de questionários específicos, como o World Health Organization Quality of Life (WHOQOL-100) (13) e o Medical Outcomes Study - Item Short-Form Health Survey (SF-36) (12, 14). Uma revisão sistemática de 12 publicações que estudaram a qualidade de vida após a alta hospitalar em sobreviventes à sepse evidenciou a clara associação entre a internação por sepse e a deterioração da qualidade de vida (10). Recentemente demonstramos o benefício da detecção precoce da sepse na probabilidade de sobrevida hospitalar de pacientes com sepse grave ou choque séptico $(15,16)$. No entanto, a sobrevida e as condições de vida dos que sobreviveram à internação não eram conhecidas. Nesse contexto, a avaliação da qualidade de vida a longo prazo desses pacientes pode ser de grande utilidade para traçar possíveis estratégias de reabilitação. Além disso, desconhecemos estudos latino-americanos que abordem o tema.

O objetivo do presente estudo foi descrever a repercussão da sepse grave e do choque séptico sobre a qualidade de vida após a alta hospitalar.

\section{MATERIAIS E MÉTODOS}

\section{Desenho do estudo, local e período}

Trata-se de um estudo controlado de delineamento transversal conduzido em duas UTIs de dois hospitais gerais localizados em Joinville, Santa Catarina, Brasil, no período de agosto de 2005 a novembro de 2007. O projeto de pesquisa foi aprovado pela Comissão de Ética em Pesquisa sob o registro de número 11.008. Todos os pacientes incluídos assinaram termo de consentimento informado.

\section{População}

Pacientes com sepse grave ou choque séptico detectados a partir do protocolo de triagem diagnóstica da CSS (17). O diagnóstico de sepse grave ou choque séptico foi realizado com base nas definições do American College of Chest Physicians/Society of Critical Care Medicine Consensus Conference Committee (ACCP/ SCCM) de 1992 (18):

- síndrome da resposta sistêmica (SIRS): presença de pelo menos dois dos seguintes dados clínico-laboratoriais: (a) febre ou hipotermia; (b) taquicardia; (c) taquipneia; (d) leucocitose ou leucopenia;

- sepse: SIRS secundária a um processo infeccioso;

- sepse grave: trata-se de um quadro de sepse complicado pela presença de pelo menos uma disfunção orgânica definida;

- choque séptico: presença de hipotensão refratária à reposição volêmica vigorosa (13).

Trata-se do arrolamento consecutivo de uma amostra "de conveniência" composta por pacientes com sepse grave ou choque séptico triados e tratados conforme orientação da CSS e que sobreviveram após dois anos da alta hospitalar $(17,19)$. Tentou-se contato telefônico com todos os sobreviventes da hospitalização. Os indivíduos encontrados constituíram o "grupo de sobreviventes" e foram entrevistados presencialmente em seus domicílios, utilizando-se o questionário SF-36.

Critérios de exclusão: menores de 18 anos, não concordância em participar do estudo, déficit cognitivo que impossibilitasse responder ao questionário, ausência de co-habitante ou vizinho de características sociodemográficas semelhantes ou impossibilidade de acesso ao domicílio do paciente.

Dados demográficos e características clínicas dos pacientes foram obtidos a partir dos registros individuais em formulário específico da CSS.

O grupo controle foi formado por um co-habitante ou vizinho com idade, sexo e escolaridade mais próxima das carac- terísticas de cada paciente e que não tivesse histórico de internação hospitalar nos últimos dois anos. Esse indivíduo respondeu ao mesmo questionário que o paciente, em entrevista pessoal. A realização do contato com o indivíduo controle seguiu os mesmos procedimentos de contato com o paciente.

\section{Questionário SF-36}

Trata-se de um instrumento genérico de avaliação da qualidade de vida composto por 36 questões, devidamente validado para a língua portuguesa e que permite avaliar aspectos de saúde física e mental, subdivididos em oito domínios: capacidade funcional, aspectos físicos, dor, estado geral de saúde, vitalidade, aspectos sociais, aspectos emocionais e saúde mental (14). O valor bruto referente a cada domínio é convertido numa escala de 0 a 100, sendo que os valores mais altos indicam melhor qualidade de vida no domínio correspondente. A pontuação de cada domínio foi calculada separadamente (não há média geral) e comparada ao grupo controle.

\section{Análise dos dados}

Variáveis clínicas e demográficas como sexo, idade, escolaridade, estado civil, renda familiar, Acute Physiology and Chronic Health Evaluation II score (APACHE II), diagnóstico de admissão, sequelas relacionadas à causa da internação na UTI, permanência na UTI e hospitalar, e mortalidade foram avaliadas. Variáveis contínuas estão apresentadas em seus valores médios \pm desvio-padrão e comparadas utilizando-se o teste $t$ de Student. As variáveis categóricas foram expressas em seus valores absolutos e relativos e comparadas com o teste qui-quadrado. A distribuição não normal dos dados relativos à pontuação dos domínios do SF-36 motivou a utilização do teste de MannWhitney, para comparação intergrupos sobre a qualidade de vida. $\mathrm{O}$ valor de $P<0,05$ foi considerado estatisticamente significativo. A estimativa da probabilidade de sobrevida em dois anos a partir da alta hospitalar foi realizada a partir da análise de Kaplan-Meier. Todas as análises foram realizadas utilizando-se os programas Number Cruncher Statistical System (NCSS) versão 2000, e o Power Analysis Statistical Software (PASS), versão 2000 (NCSS, Kaysville, Utah), ou o 
programa Statistical Package for the Social Sciences, versão 13.0 (SPSS Inc, Chicago, Illinois).

\section{RESULTADOS}

Foram identificados 217 pacientes com sepse grave ou choque séptico, dos quais $112(51,6 \%)$ sobreviveram à internação. Destes, $27(24,1 \%)$ faleceram após a alta e $63(56,3 \%)$ foram confirmados como sobreviventes (figura 1). Vinte e dois $(19,6 \%)$ não foram localizados e, por essa razão, censurados na análise de sobrevida. A probabilidade acumulada de sobrevida associada à sepse grave e choque séptico foi de 41,02\% (80/195) em 6 meses, 37,4\% (73/195) em 12 meses, 34,3\% (67/195) em 18 meses e 32,3\% (63/195) em 24 meses.

Dentre os sobreviventes, 27 foram excluídos da análise de qualidade de vida pelas seguintes razões: não foram encontrados no endereço fornecido no contato telefônico inicial (No. = 8); negaram-se a participar do estudo (No. $=3)$; residiam em outro município (No. = 12); ou porque não havia indivíduo controle correspondente (No. =4). Ao final, 36 indivíduos constituíram o grupo de sobreviventes e responderam às perguntas do questionário SF-36 (figura 1). O tempo médio entre a alta hospitalar e a entrevista foi de $35 \pm 6,5$ meses (mínimo: 24 meses; máximo: 51 meses).

A tabela 1 exibe as características clínicas dos sobreviventes por ocasião da internação. Idade, sexo, estado civil e escolaridade do grupo de sobreviventes foram semelhantes aos do grupo controle.

A comparação entre os grupos demonstra menor pontuação no escore de qualidade de vida do grupo de sobreviventes em relação ao grupo controle em sete dos oito domínios avaliados. Não houve diferença estatisticamente significativa no domínio "aspectos emocionais". Os sumários de saúde física e de saúde mental foram diferentes entre os grupos (tabela 1).

No momento da entrevista, 11 pacientes tinham sequelas associadas ao diagnóstico de internação e que poderiam afetar a qualidade de vida (sequela de trauma: 4; sequela de meningite: 1 ; colostomia: 2; câncer: 2; sequela de acidente vascular encefálico: 2 ). A supressão desses indivíduos da análise global não modificou os resultados: capacidade funcional $(59 \pm 32$ versus $91 \pm 18$; $P<0,001)$, vitalidade $(48 \pm 13$ versus
FIGURA 1. Distribuição dos indivíduos incluídos no estudo

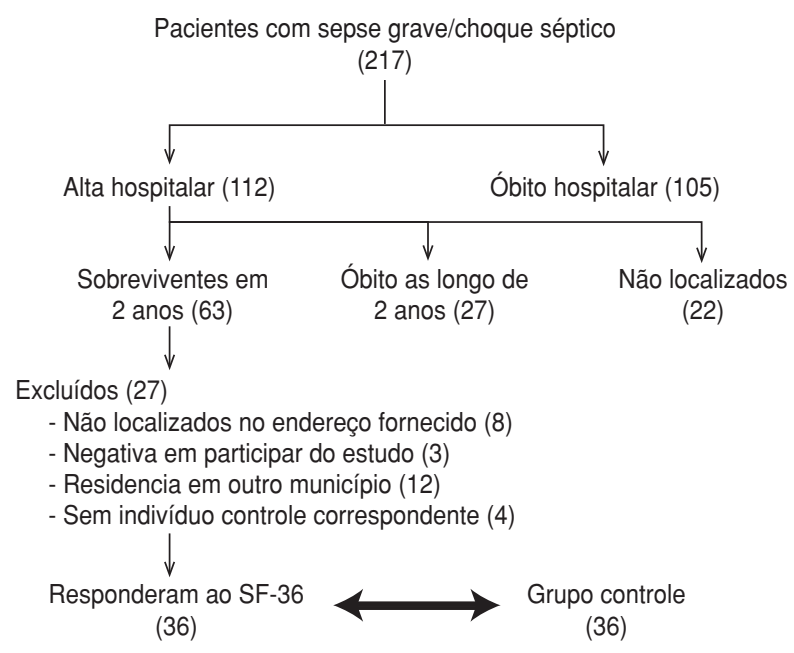

SF-36: Short Form-36.

TABELA 1. Características clínico-demográficas e pontuação dos domínios do SF-36 e pneumonia observadas entre o grupo de sobreviventes e grupo controle

\begin{tabular}{|c|c|c|c|}
\hline Característica & $\begin{array}{l}\text { Sobreviventes } \\
\qquad(n=36)\end{array}$ & $\begin{array}{l}\text { Controle } \\
(n=36)\end{array}$ & Valor de $P$ \\
\hline Idade, anos & $46,6 \pm 18,8$ & $43,5 \pm 16,1$ & 0,55 \\
\hline Sexo masculino, №. (\%) & $17(47,3)$ & $11(52,7)$ & 0,14 \\
\hline APACHE II & $17 \pm 7$ & - & - \\
\hline Permanência hospitalar, dias & $44,4 \pm 46,6$ & - & - \\
\hline Permanência na UTI, dias & $17,6 \pm 24,2$ & - & - \\
\hline Sepse grave, №. (\%) & $16(44,5)$ & - & - \\
\hline Choque séptico, No. (\%) & $20(55,5)$ & - & - \\
\hline \multicolumn{4}{|l|}{ Foco infeccioso } \\
\hline Abdominal, No. (\%) & $9(25)$ & - & - \\
\hline Meningite, №. (\%) & $2(5,5)$ & - & - \\
\hline PNM, No. (\%) & $13(36,1)$ & - & - \\
\hline Partes moles, No. (\%) & $3(8,3)$ & - & - \\
\hline Urinário, №. (\%) & $3(8,3)$ & - & - \\
\hline Indeterminado, №. (\%) & $6(16,6)$ & - & - \\
\hline \multicolumn{4}{|l|}{ Estado civil } \\
\hline Solteiro, No. (\%) & $10(27,8)$ & $13(36,1)$ & 0,44 \\
\hline Casado, No. (\%) & $20(55,6)$ & $19(52,8)$ & 0,81 \\
\hline Divorciado/viúvo, No. (\%) & $6(16,6)$ & $4(11,1)$ & 0,49 \\
\hline \multicolumn{4}{|l|}{ Escolaridade } \\
\hline Ensino fundamental, No. (\%) & $19(52,8)$ & $14(52,8)$ & 0,23 \\
\hline Ensino médio, №. (\%) & $14(38,9)$ & $13(36,1)$ & 0,80 \\
\hline Ensino superior, №. (\%) & $3(8,3)$ & $9(11,1)$ & 0,06 \\
\hline \multicolumn{4}{|l|}{ Domínios do SF-36 } \\
\hline Capacidade funcional & $62 \pm 33$ & $93 \pm 16$ & $<0,001$ \\
\hline Vitalidade & $51 \pm 14$ & $60 \pm 14$ & $<0,008$ \\
\hline Saúde mental & $63 \pm 22$ & $75 \pm 18$ & $<0,03$ \\
\hline Dor & $57 \pm 27$ & $76 \pm 17$ & $<0,001$ \\
\hline Estado geral de saúde & $55 \pm 19$ & $66 \pm 15$ & $<0,004$ \\
\hline Aspectos físicos & $67 \pm 45$ & $85 \pm 34$ & $<0,05$ \\
\hline Aspectos sociais & $76 \pm 27$ & $89 \pm 16$ & $<0,05$ \\
\hline Aspectos emocionais & $72 \pm 43$ & $89 \pm 29$ & 0,11 \\
\hline Sumário de saúde física & $62 \pm 20$ & $78 \pm 12$ & $<0,004$ \\
\hline Sumário de saúde mental & $67 \pm 30$ & $82 \pm 20$ & $<0,03$ \\
\hline
\end{tabular}

APACHE II: Acute Physiology and Chronic Health Evaluation II; variáveis contínuas estão expressas em média \pm desviopadrão. Aplicou-se o teste do qui-quadrado para análise de variáveis categóricas, o teste $t$ de Student para análise de variáveis com distribuição normal, e o teste de Mann-Whitney para variáveis de distribuição não normal (comparação intergrupos da qualidade de vida). Valores de $P<0,05$ foram considerados estatisticamente significativos.

UTI: Unidade de Terapia Intensiva; PNM: pneumonia; SF-36: Short Form-36. 
TABELA 2. Mudanças nas condições de saúde, trabalho e renda do grupo de sobreviventes em relação ao período anterior à internação

\begin{tabular}{lc}
\hline & No. (\%) \\
\hline Comparado há 1 ano como está sua saúde hoje? & 0 \\
Muito melhor & $15(41,7)$ \\
Pouco melhor & $15(41,7)$ \\
Igual & $6(16,6)$ \\
Pouco pior & 0 \\
Muito pior & $11(30,5)$ \\
Com relação ao trabalho & $11(30,5)$ \\
$\quad$ Diminuiu o tempo de dedicação & $13(36,1)$ \\
Realizou menos tarefas & $13(36,1)$ \\
Apresentou limitação & $10(27,8)$ \\
Teve dificuldade na realização & $23(63,9)$ \\
Renda no último ano & $3(8,3)$ \\
Diminuiu & \\
Não diminuiu & \\
Não possui &
\end{tabular}

$59 \pm 14 ; P<0,04)$, saúde mental $(60 \pm 24$ versus $75 \pm 17 ; P<0,02)$, dor $(50 \pm 26$ versus $76 \pm 16 ; P<0,004)$, estado geral de saúde $(54 \pm 18$ versus $67 \pm 13 ; P<0,02)$, aspectos físicos $(60 \pm 45$ vesus $87 \pm 34$; $P<0,02)$ e aspectos sociais $(70 \pm 28$ versus $90 \pm 16 ; P<0,003)$ e "aspectos emocionais" (64 \pm 46 versus $85 \pm 33 ; P=0,09)$.

Em relação ao período anterior à internação, houve relato de limitações importantes para realização das atividades laborais, diminuição da renda e impressão subjetiva de comprometimento global da saúde por parte dos sobreviventes à sepse (tabela 2).

\section{DISCUSSÃO}

Os resultados deste estudo demonstram que os sobreviventes da sepse grave e/ou choque séptico estão sujeitos a grave comprometimento da qualidade de vida na maior parte dos aspectos físicos e mentais quando comparados a uma população controle, e reforçam a ideia de que a maior probabilidade de óbito desses pacientes perdura mesmo após alta hospitalar.

Qualidade de vida e mortalidade após a alta hospitalar de pacientes graves são focos de intensa pesquisa nos últimos anos. No que diz respeito à qualidade de vida de pacientes com sepse grave, há um número cada vez maior de publicações. Na América Latina, no entanto, este é o primeiro registro de qualidade de vida após alta hospitalar que avalia tal população específica com o questionário SF-36. Nossos resultados, de forma geral, são semelhantes aos de outras publicações que, em sua maioria, demonstram redução de qualidade de vida nos mais diversos aspectos (10). Cabral et al. avaliaram a qualidade de vida pós-alta hospitalar a partir da aplicação da escala de Karnofsky em todos os pacientes graves que receberam alta da UTI. Houve preservação da qualidade de vida na análise global dessa população e também no subgrupo de pacientes sépticos. Os resultados provavelmente foram influenciados pela utilização da escala de Karnofzky, que se limita a avaliar o grau de autonomia/dependência individual baseada essencialmente na capacidade de realizar autocuidados. Ao contrário, o questionário SF-36 avalia diversos domínios que influenciam a qualidade de vida. A ênfase da escala de Karnofsky sobre a capacidade de realização de autocuidados explica a redução de qualidade de vida encontrada exclusivamente no subgrupo de pacientes com acidente vascular encefálico (20).

Apesar do melhor momento para avaliação de qualidade de vida em pacientes graves que sobreviveram à internação hospitalar não estar bem estabelecido, a maioria dos estudos sobre esse tema faz a avaliação entre o $6^{\circ}$ e o $12^{\circ}$ mês após a alta $(10,21)$. A revisão sistemática publicada por Winters et al. avaliou 12 estudos de qualidade de vida em pacientes sépticos e o período de seguimento dos pacientes foi menor que 1 ano em sete dessas 12 publicações ( 3 a 12 meses) (10), diferindo do nosso estudo, no qual o tempo médio entre alta e entrevista foi de $35 \pm 6,5$ meses. Nesse aspecto, entendemos que um período maior de observação pode nos informar de forma mais precisa as repercussões da sepse grave e do choque séptico sobre a saúde dos sobreviventes. Curtos períodos de observação não permitem avaliar alguns efeitos da doença sobre a saúde a longo prazo (10).

Há vários métodos de aferição de qualidade de vida pós-alta hospitalar em pacientes graves, dentre os quais podemos citar o SF-36 (58,4\% dos estudos), Nottingham Health Profile (NHP), EuroQol-5D (EQ-5D), RAND 36-Item Health Survey (RAND-36) e o Patrick's Perceived Quality of Life Scale (PQoL) (21). Dos 12 estudos que avaliaram qualidade em sobreviventes da sepse grave, o SF-36 foi utilizado em seis, o EuroQol-5D em três, WHO score em um, Modified quality of life score (Modified Qol) em um e Karnovsky em um (10). Pacientes que sobreviveram ao episódio séptico apresentam diminuição da qualidade de vida independentemente do método de aferição empregado (10). Não há consenso sobre qual o melhor instrumento para medir qualidade de vida em pacientes de UTI e/ou com sepse. Optamos pela utilização do SF-36, por se tratar de um questionário de simples aplicação, amplamente utilizado para avaliar a qualidade de vida de diversas populações e validado para avaliação da qualidade de vida de pacientes sépticos $(10,12,21)$.

A aplicação de questionários para avaliação de qualidade de vida após alta hospitalar deveria, idealmente, ser precedida de entrevista que contemplasse a fase pré-doença. Karlson et al., utilizando o questionário EQ-5D, observaram que a qualidade de vida de 98 pacientes com sepse grave foi comprometida de forma significativa após 17 meses da alta em relação ao período pré-internação hospitalar (22). Hofhuis et al. observaram prospectivamente a ocorrência de um forte declínio da qualidade de vida durante a internação na UTI seguida de recuperação gradual, mas incompleta, em vários domínios ao longo de 6 meses (23). No entanto, a comparação com o período pré-hospitalização é permeada de grande dificuldade prática. Boa parte das informações sobre morbidade prévia não são confiáveis, considerando que a possível presença do viés de tempo decorrente dos longos períodos após a alta hospitalar pode acarretar falsas percepções do passado remoto nos pacientes.

No presente estudo, apesar do resultado das entrevistas pós-alta não ter sido comparado à fase pré-doença, houve comparação com o grupo controle constituído de indivíduos com características sociodemográficas semelhantes às dos 
sobreviventes da sepse. Essa particularidade, embora aborde apenas superficialmente tais características, diferencia nosso estudo de outros que comparam os dados da média populacional $(12,22-$ 24), permitindo estimar de forma mais apropriada o impacto da sepse grave sobre a qualidade de vida. Por outro lado, não comparamos a população séptica com portadores de outras doenças.

O declínio de qualidade de vida pósalta hospitalar de pacientes sépticos observado no nosso e em outros estudos é similar ao que se observa em pacientes com lesão pulmonar aguda (LPA) ou síndrome do desconforto respiratório agudo (SDRA), doença pulmonar obstrutiva crônica (DPOC) e insuficiência cardíaca congestiva (ICC) (10, 25-27), assim como em outras doenças agudas graves $(7,28-32)$. Considerando que a sepse é uma das principais complicações dos pacientes graves e a principal causa de LPA/SDRA, não é possível determinar se os efeitos observados a longo prazo são decorrência exclusiva da sepse, da LPA/SDRA ou da doença aguda subjacente (10).

Weycker et al. observaram que, além da mortalidade, também os custos diretos são elevados nos anos que se seguem à hospitalização (33). Embora a avaliação de custo direto não tenha sido realizada aqui, é possível fazer algumas considerações sobre o custo indireto, que compõe cerca de $70 \%$ do custo total relacionado à sepse $(2,34,35)$. De acordo com o recente estudo de Koenig et al., grande parte desse custo está ligado à abreviação de anos de vida produtivos associada à alta mortalidade dessa doença (35). Considerando a baixa média de idade (46,6 $\pm 18,8$ anos de idade) dos 36 pacientes avaliados no presente estudo, podese inferir que não apenas a mortalidade, mas também a deterioração da qualidade de vida, afeta a capacidade laboral de pacientes que desenvolveram sepse, tendo implicações socioeconômicas. Esse aspecto é reforçado quando observamos que $30,5 \%$ dos pacientes relataram ter limitações em relação à sua capacidade produtiva e que em $27,8 \%$ houve comprometimento da renda pessoal do último ano (tabela 2). Desse modo, o custo para a sociedade derivado da perda da produtividade individual e não oriunda apenas da mortalidade, mas também do comprometimento da qualidade de vida. Algumas análises econômicas disponíveis mostram que a padronização dos regimes terapêuticos reduz a mortalidade e o custo direto hospitalar relacionado à sepse $(1,36-38)$. Nesse sentido, é possível que a adoção de programas de reabilitação e reintegração desses indivíduos possa acelerar a recuperação da capacidade produtiva e reduzir o custo indireto da sepse à sociedade.

É provável que a gravidade das alterações celulares agudas da sepse seja responsável pela deterioração das funções orgânicas ao longo do tempo, afetando a qualidade de vida dos sobreviventes a longo prazo. Do mesmo modo, a gravidade do episódio séptico está associada ao maior risco de morte após a alta hospitalar $(2,10)$. Quartin et al. observaram que a vida de um paciente que sobrevive à sepse é reduzida em 2,6 anos (2). Esses dados são corroborados pela revisão sistemática publicada em 2010 por Winters et al., na qual se observa que a mortalidade associada ao choque séptico continua elevada mesmo nos meses e anos que se seguem à alta hospitalar nos 30 estudos analisados. Em todos os estudos que avaliaram a mortalidade relacionada à sepse até um ano após a alta, foram observadas taxas de mortalidade de $21,5 \%$ a $71,9 \%$ (10). Entre os estudos observacionais (8 em 17), a mortalidade em um ano variou de 11 a 42,5\% (10). Os dois estudos em que o seguimento foi de dois anos a mortalidade global ao final desse período era de 44,9 e $67 \%$ (a mortalidade hospitalar foi de 28,3 e $40 \%$ respectivamente) (10). Esses resultados, de forma geral, assemelham-se aos do nosso estudo, em que a mortalidade hospitalar foi de $48,4 \%$, enquanto a mortalidade global, em um ano, foi de $56,2 \%$, e, em dois anos, de $60,8 \%$. A semelhança se mantém quando se avalia a taxa de mortalidade ao final de um ano dos que sobreviveram à internação. Essa taxa varia de 7 a $43 \%$ na literatura (10).

Há algumas limitações que devem ser consideradas. Em razão das características do formulário original de triagem dos pacientes incluídos pelo protocolo da CSS (17), não houve registro de comorbidades existentes por ocasião da internação hospitalar. Assim, a análise dessas comorbidades não foi possível. Outro aspecto a ser levado em conta é que os pacientes entrevistados não foram comparados com pacientes graves não sépticos, fator que poderia auxiliar na definição da participação de comorbidades prévias ou decorrentes da estada na UTI na deterioração da qualidade de vida. Essa preocupação é reforçada pelos resultados de Cook et al. que, apesar da redução de qualidade de vida em relação à população normal, não houve diferenças em relação à população não séptica internada na UTI, embora o período de observação tenha se limitado a três meses (24). Os critérios utilizados para assemelhar os grupos do nosso estudo (um co-habitante ou vizinho com a idade, sexo e escolaridade mais próxima das características de cada paciente e que não tivesse histórico de internação hospitalar nos últimos dois anos), embora permitam levar em conta o contexto socioeconômico, fazem a avaliação desse aspecto apenas de maneira superficial. Por fim, o longo período decorrido até a realização da entrevista pode ter influenciado os resultados, tendo em vista o potencial somatório de comorbidades ao longo desse período.

A escassez de recursos financeiros e a demanda crescente por tecnologias de ponta para tratamento de pacientes graves tornam o conhecimento sobre a repercussão da sepse na mortalidade e qualidade de vida aspecto essencial para o planejamento de ações de saúde e destinação de recursos. A baixa média de idade da população estudada e os anos de vida produtiva comprometidos em consequência da síndrome séptica reforçam a importância de tais ações, assim como a ideia de que os desfechos estudados não podem se resumir à contabilização de mortos e vivos $(12,21)$. Apesar dos resultados enfatizarem achados de outras publicações, devemos destinar esforços para transpor as limitações de estudos dessa natureza. É fundamental a busca por alternativas metodológicas que permitam avaliar o efeito isolado da síndrome séptica sobre a qualidade de vida, suplantando a influência da subjetividade dos escores de qualidade de vida e de afecções coexistentes.

Podemos concluir que a sepse grave, além de estar associada a altas taxas de mortalidade hospitalar, pode resultar em comprometimento significativo da qualidade de vida daqueles que sobrevivem à internação, bem como comprometer a probabilidade de sobrevida a longo prazo. 


\section{REFERÊNCIAS}

1. Angus DC, Linde-Zwirble WT, Lidicker J, Clermont G, Carcillo J, Pinsky MR. Epidemiology of severe sepsis in the United States: analysis of incidence, outcome and associated costs of care. Crit Care Med. 2001;29(7): 1303-10.

2. Quartin A, Schein RMH, Kett DH, Peduzzi PN. Magnitude and duration of the effect of sepsis on survival. JAMA. 1997;277(13):1058-63.

3. Brun-Buisson C, Doyon F, Carlet J, Dellamonica P, Gouin F, Lepoutre A, et al. Incidence, risk factors and outcome of severe sepsis and septic shock in adults: a multicenter, prospective study in intensive care units. French ICU Group for Severe Sepsis. JAMA. 1995;274(12):968-74.

4. Office of Planning and Extramural Programs and Hospital Care Statistics, Division of Health Care Statistics, National Center for Health Statistics. Increase in national hospital discharge survey rates for septicemia-United States, 1979-1987. MMWR Morb Mortal Wkly Rep. 1990;39:31-4.

5. Kochanek KD, Hudson BL. Division of vital statistics: advance report of final mortality statistics, 1992. Mthly Vital Stat Rep. 1995;43.

6. Sales JA Jr, Lobo SMA, Hatum R, Dias FD, Macedo GL, Costa JI et al. Sepse Brasil: estudo epidemiológico da sepse em unidades de terapia intensiva brasileiras. Rev Bras Ter Intensiva. 2006;18:9.

7. Silva E, Pedro Mde A, Sogayar AC, Mohovic $\mathrm{T}$, Silva CL, Janiszewski M, et al. Brazilian Sepsis Epidemiological Study (BASES study). Crit Care Med. 2004;8(4):R251-60.

8. Levy MM, Dellinger RP, Townsend SR, Linde-Zwirble WT, Marshall JC, Bion J, et al. The Surviving Sepsis Campaign: results of an international guideline based performance improvement program targeting severe sepsis. Crit Care Med. 2010;38(2):367-74.

9. Latin American Sepsis Institute [Internet]. Campanha Sobrevivendo à Sepse. Available from: <http:/ / www.sepsisnet.org/PDF/ Relatorio\%20Nacional\%20SSC.pdf> Cited 20 March 2012.

10. Winters BD, Eberlein M, Leung J, Needham DM, Pronovost PJ, Sevransky JE. Long-term mortality and quality of life in sepsis: A systematic review. Crit Care Med. 2010;38(5):1276-83.

11. Teles JM, Silva E, Westphal G, Filho RC, Machado FR. Surviving sepsis campaign in Brazil. Shock. 2008;30(Suppl 1):47-52.

12. Heyland DK, Hopman, Coo H, Tranmer J, McColl MA. Long-term health-related quality of life in survivors of sepsis. Short Form 36: A valid and reliable measure of health-related quality of life. Crit Care Med. 2000;28(11): 3599-605.

13. Fleck MP, Chachamovich E, Trentini CM. [WHOQOL-OLD Project: method and focus group results in Brazil]. Rev Saude Publica. 2003;37(6):793-9. Article in Portuguese.

14. Ciconelli RM, Ferraz MB, Santos W, Meinão I, Quaresma MR. Tradução para a língua portu- guesa e validação do questionário genérico de avaliação de qualidade de vida SF-36 (Brasil SF-36). Rev Bras Reumatol. 1999;39:143-50.

15. Westphal GA, Koenig A, Caldeira Filho M, Feijó J, de Oliveira LT, Nunes F, et al. Reduced mortality after the implementation of a protocol for the early detection of severe sepsis. J Crit Care. 2011;26(1):76-81.

16. Westphal GA, Feijó J, de Andrade PS, Trindade L, Suchard C, Monteiro AG, et al. Estratégia de detecção precoce e redução de mortalidade na sepse grave. Rev Bras Ter Intensiva. 2009;21(2):113-23.

17. American Sepsis Institute [Internet]. Campanha Sobrevivendo à Sepse. Available from: http:/ / www.sepsisnet.org/PDF/sugestao \%20de\%20ficha\%20de\%20triagem.pdf Cited 20 March 2012.

18. Bone RC, Balk RA, Cerra FB, Dellinger RP, Fein AM, Knaus WA, et al. Definitions for sepsis and organ failure and guidelines for the use of innovative therapies in sepsis. The ACCP/ SCCM Consensus Conference Committee. American College of Chest Physicians/ Society of Critical Care Medicine. Chest. 1992; 101(6):1644-55

19. American Sepsis Institute [Internet]. Campanha de Sobrevivência à Sepse. Protocolo clínico. Available from: http://www.sepsis net.org/PDF/sugestao\%20de\%20protocolo\% 20de\%20tratamento.pdf Cited 20 March 2012.

20. Cabral RC, Teixeira C, Oliveira RP, Hass JS, Azzolin KO. Avaliação da mortalidade e qualidade de vida dois anos após a alta do CTI: dados preliminares de uma coorte prospective. Rev Bras Ter Intensiva. 2009;21(1):18-24.

21. Oeyen SG, Vandijck DM, Benoit DD, Annemans L, Decruyenaere JM. Quality of life after intensive care: a systematic review of the literature. Crit Care Med. 2010;38(12): 2386-400.

22. Karlsson S, Ruokonen E, Varpula T, Ala-Kokko TI, Pettilä V; Finnsepsis Study Group. Long-term outcome and qualityadjusted life years after severe sepsis. Crit Care Med. 2009;37(4):1268-73.

23. Hofhuis JG, Spronk PE, van Stel HF, Schrijvers AJ, Rommes JH, Bakker J. The impact of severe sepsis on health-related quality of life: A long-term follow-up study. Critical Care and Trauma. 2008;107(6):1957-64

24. Cook WT, Eddleston JM, Conway D, Streets J. Quality of life in ICU survivors with severe sepsis who received activated protein $\mathrm{C}$. Crit Care Med. 2003;7(Suppl 2):P023

25. Rubenfeld GD, Caldwell E, Peabody E, Weaver J, Martin DP, Neff M, et al. Incidence and outcomes of acute lung injury. N Engl J Med. 2005;353(16):1685-93.

26. Hudson LD, Milberg JA, Anardi D, Maunder RJ. Clinical risks for development of the acute respiratory distress syndrome. Am J Respir Crit Care Med. 1995;151(2 Pt 1):293-301.

27. Granja C, Dias C, Costa-Pereira A, Sarmento A. Quality of life of survivors from severe sepsis and septic shock may be similar to that of others who survive critical illness. Crit Care Med. 2004;8(2):R91-8.

28. Dowdy DW, Eid MP, Sedrakyan A, MendezTellez PA, Pronovost PJ, Herridge MS, et al. Quality of life in adult survivors of critical illness: A systematic review of the literature. Intensive Care Med. 2005;31(5):611-20.

29. Hopkins RO, Jackson JC. Long-term neurocognitive function after critical illness. Chest. 2006;130(3):869-78

30. Gunther ML, Jackson JC, Ely EW. The cognitive consequences of critical illness: practical recommendations for screening and assessment. Crit Care Clin. 2007;23(3):491-506.

31. Rattray JE, Johnston M, Wildsmith JA. Predictors of emotional outcomes of intensive care. Anaesthesia. 2005;60(11):1085-92.

32. Longo CJ, Heyland DK, Fisher HN, Fowler RA, Martin CM, Day AG. A long-term followup study investigating health-related quality of life and resource use in survivors of severe sepsis: comparison of recombinant human activated protein $\mathrm{C}$ with standard care. Critical Care. 2007;11(6):R128.

33. Weycker D, Akhras KS, Edelsberg J, Angus DC, Oster G. Long-term mortality and medical care charges in patients with severe sepsis. Crit Care Med. 2003;31:2316-22.

34. Ware JE. The SF-36 health survey. In: Spilker B. Quality of life and pharmacoeconomics in clinical trials. Philadelphia: Lippincott-Raven; 1996. Pp. 281-6.

35. Koenig A, Picon PD, Feijó J, Silva E, Westphal GA. Estimativa do impacto economico da implantação de um protocolo hospitalar para detecção e tratamento precoce da sepse grave em hospitais públicos e privados do sul do Brasil. Rev Bras Ter Intensiva. 2010; 22(3):213-9.

36. Sogayar AM, Silva E, Cal RG, Beer I, Akamine N, Safi J Jr, et al. The Costs Group. What are the direct costs of sepsis treatment in Brazilian ICUs? Crit Care. 2005;9(Suppl 2):P112.

37. Schorr AF, Micek ST, Jackson WL Jr, Kollef $\mathrm{MH}$. Economic implications of an evidencebased sepsis protocol: can we improve outcomes at lower costs? Crit Care Med. 2007; 35(5):1257-62.

38. Sogayar AM, Machado FR, Rea-Neto A, Dornas A, Grion CM, Lobo SM, et al. A multicentere, prospective study to evaluate costs of septic patients in Brazilian intensive care units. Pharmacoeconomics. 2008;26(5):425-34.

Manuscrito recebido em 31 de março 2011. Aceito em versão revisada em 8 de março de 2012. 
ABSTRACT Objective. Describe the impact of severe sepsis and septic shock on patients' quality of life following hospital discharge.

\section{Analysis of quality of life following hospital discharge among survivors of severe sepsis and septic shock}

Methods. A controlled study conducted in two general hospitals of Joinville, Santa Catarina, Brazil, of in-patients with severe sepsis or septic shock during the period of August 2005 through November 2007. The patients were contacted by telephone between June and November 2009. The study group responded to Short Form-36, a questionnaire on the quality of life, two years after being discharged from hospital. The questionnaire was also answered by a control group composed of people who lived at the same residence as the study subjects, had no recent hospitalization, and were close in age.

Results. Of 217 patients with severe sepsis or septic shock, $112(51.6 \%)$ survived hospitalization. The survival rate after hospital discharge was $41.02 \%$ at 180 days, $37.4 \%$ at one year, $34.3 \%$ at 18 months, and $32.3 \%$ in two years. Thirty-six survivors responded to Short Form-36. There were declines in the quality of life for survivors $($ No. = 36) in comparison to the control group $(\mathrm{No} .=36)$ in the following areas: physical functioning ( $59 \pm 32$ versus $91 \pm 18 ; P<0.001)$, vitality $(48 \pm 13$ versus $59 \pm 14$; $P<0.008)$, mental health ( $48 \pm 13$ versus $59 \pm 14 ; P<0.03)$, bodily pain $(50 \pm 26$ versus $76 \pm 16 ; P<0.001)$, general health perceptions $(53 \pm 18$ versus $67 \pm 13 ; P<0.004)$, physical role functioning $(67 \pm 45$ versus $85 \pm 34 ; P<0.05)$, and social role functioning $(70 \pm 28$ versus $90 . \pm 16 ; P<0.05)$.

Conclusions. Severe sepsis or septic shock can result in significant negative effects on the quality of life, in addition to reducing long-term survival probability.

Key words Sepsis; shock, septic; quality of life; mortality; Brazil. 